\author{
MONIKA KOWALONEK-JANCZAREK \\ Uniwersytet im. Adama Mickiewicza w Poznaniu \\ monika@amu.edu.pl \\ ORCID: 0000-0002-9230-1948
}

\title{
Englisch als Lingua franca versus andere Sprachen im Bildungswesen: Polen und Japan im Vergleich
}

\section{English as a lingua franca versus other languages in Polish and Japanese education systems}

\begin{abstract}
The purpose of this study is to compare Poland's and Japan's foreign language policies in preschool, elementary, lower/upper secondary and higher education and shed light on the aspect of multilingualism in this milieu as well. Based on secondary data (ministerial ordinances, governmental reports, curricula) and literature knowledge, the paper provides a comparative overview of the Polish and Japanese contexts which differ in a strong way. While Poland's foreign language policy is mainly based on the objectives of the EU's policy according to which every European citizen should master two other languages in addition to his or her mother tongue, the Japanese government's policy aims at improving English education.
\end{abstract}

KEYWORDS: foreign language policy, Poland, Japan, multilingualism, EU, ASEAN, lingua franca.

SCHLÜSSELWORTE: Fremdsprachenpolitik, Polen, Japan, Mehrsprachigkeit, EU, ASEAN, Lingua franca.

\section{EINLEITUNG}

Die Rahmenbedingungen des Fremdsprachenlehrens werden in großem Maße von bildungs- sowie sprachpolitischen Entscheidungen beeinflusst. Die Sprachenpolitik, einst wurde sie in der Forschung häufig übersehen, 
wird heutzutage vermutlich von allen Staaten verwendet, sei es in dem MaBe in welcher Sprache kommuniziert werden soll oder sei es auch, welche Sprache in den jeweiligen Bildungsinstitutionen erlernt werden soll, auch wenn solche Entscheidungen zumeist nicht Sprachenpolitik genannt werden (Ammon 2010: 636). Die Aufgabe, Fremdsprachen unter institutionellen Bedingungen zu lehren, erfüllen $u$. a. Kindergärten, Schulen oder Universitäten, die in den vom Staat definierten Vorgaben für Unterricht und Erziehung als Richtlinien vorgelegt werden. Bei der Fremdsprachenvermittlung spielt allerdings oft nicht nur der bildungspolitische, sondern auch der ideologische Kontext eine Rolle. In Europa ist die Förderung der Fremdsprachenkenntnisse von mindestens zwei Ideen geprägt: zum einen von der Mehrsprachigkeit, sowie zum anderen von der Ökolinguistik. Da die Vielfalt der Sprachen und Kulturen die Besonderheit Europas ausmacht, wundert es nicht, dass hierbei die Verknüpfung der Sprachenpolitik mit dem Mehrsprachigkeitskonzept einerseits, sowie mit der Förderung der ökolinguistischen Ideologie andererseits, die es sich zur Aufgabe gemacht hat, natürliche Objekte und derer Umwelt zu schützen, so eng miteinander verbunden sind. Bereits im Jahre 1958 legte die Verordnung Nr. 1 zur Sprachenfrage für die Europäische Wirtschaftsgemeinschaft den Grundsatz der Mehrsprachigkeit fest, indem die Amtssprache eines jeden Mitgliedslandes automatisch zur Amtssprache der Union wurde. Heute bekennt sich die Europäische Union zu ihren 28 Amtssprachen und spricht sich gegen die Dominanz einer Lingua franca aus. Des Weiteren ist die Sprachenpolitik Europas insofern von großer Relevanz, als sie zudem laut Pfeiffer (2005: 309) „der Vertiefung der europäischen Integration” dienen soll, sowie „die unumgängliche Voraussetzung dafür" ist. Auch in Asien gibt es Versuche, die Region nach dem Vorbild der EU wirtschaftlich zu integrieren. 1967 wurde die Organisation ASEAN11 (Association of Southeast Asian Nations) gegründet, welche 1997 um die Staaten China, Japan und Südkorea erweitert wurde (ASEAN-plusDrei). Die Kooperation umfasst mittlerweile verschiedene Bereiche wie beispielsweise die Sicherheit, den Handel, den Tourismus, die Energie, sowie die Bildungswissenschaft. Sie versteht sich als Diskussionsforum. In diesem Zusammenhang verweisen Kirkpatrick und Liddicoat (2017: 2) darauf, dass auch die Sprachenpolitik als ein wichtiges politisches Thema anzusehen ist, denn: „This regional grouping thus represents a key element of Asian collaboration and its member nations have many similarities that justify considering it as a grouping for discussing language education policy“.

${ }^{1}$ Damit sind die Länder Brunei, Kambodscha, Indonesien, Laos, Malaysia, Myanmar, Philippinen, Singapur, Thailand und Vietnam gemeint. 
Der einleitende kurz gefasste und im Weiteren skizzierte Kontext führt zu der Frage, welche Ausformung ein zukunftsorientiertes Fremdsprachenunterrichtskonzept im Bildungswesen verschiedener Länder in einer zunehmend globalisierten Welt des 21. Jahrhunderts haben kann. Ziel dieses Beitrags wird es sein, eine Bestandsaufnahme über die aktuelle Situation im Bereich des Fremdsprachenunterrichts in Polen und Japan vorzunehmen. Insofern sind die folgenden unten aufgeführten Fragen meinerseits als Leitfragen der Studie anzusehen:

- In welcher Regelaltersstufe beginnen Schüler/-innen in Polen sowie Japan im Vergleich mit einer Fremdsprache?

- Welche- und wie viele Sprachen genau werden im Bildungswesen der jeweiligen Länder angeboten? (Es ist hierbei zu erwähnen, dass hier sowohl die Stufen vom Vor- bis zum Hochschulbereich gemeint sind2).

- Welche spezifischen Schwerpunktbereiche lassen sich in den jeweiligen Richtlinien/Regelungen ${ }^{3}$ für den Fremdsprachenunterricht in den jeweiligen Ländern erkennen?

Der Artikel versteht sich als Anerkennung zahlreicher Errungenschaften von Prof. Dr. Waldemar Pfeiffer auf dem Gebiet der Glottodidaktik im Allgemeinen sowie Fremdsprachenpolitik im Besonderen.

\section{FREMDSPRACHENPOLITIK}

Vor einer Auseinandersetzung mit der sprachpolitischen Situation Polens sowie zum anderen Japans, ist zu klären, was man unter dem Terminus Sprachenpolitik genauer verstehen kann. In der Fachliteratur werden oftmals die Termini Sprach- und Sprachenpolitik als Synonyme erfasst, was ein Blick in die linguistischen Fachlexika bestätigt (Bußmann 2002: 628; Krumm 2010: 298). Gegenüber diesen kompakten Definitionen findet man bei anderen Autoren auch eine differenziertere Variante, in der zwischen Sprachpolitik und Sprachenpolitik unterschieden wird. So führt Witt (2001: 23) ein quantitatives Kriterium ein (in Anlehnung an Bochmann (1993: 13) und Stark (1997: 28), die beide die Ansicht vertreten, dass Sprachpolitik sich auf eine Sprache, während dessen sich hingegen die Sprachenpolitik auf mehr als eine Sprache bezieht. Eine noch anderweitige Unterteilung nimmt Pfeiffer (2004: 189-192) vor, der zufolge Sprachpolitik im Politikbereich der jeweiligen Muttersprache

\footnotetext{
2 Vorauszuschicken ist an dieser Stelle, dass bilinguale sowie Berufs-, Fachober- und Förderschulen nicht berücksichtigt werden.

${ }^{3}$ Sofern ich nicht aus den jeweiligen Dokumenten zitiere, werden die Termini Richtlinienregelungen, Richtlinien, Rahmenprogramme, Rahmenlehrprogramme und Programmgrundlagen in meinem Beitrag synonym verwendet.
} 
(z. B. ihre Normierung und Pflege) angewendet wird. Die Sprachenpolitik, die den Gegenstand des vorliegenden Beitrags bildet, versteht Pfeiffer hingegen als eine Politik im Bereich des Fremdsprachenunterrichts. Dabei unterscheidet Pfeiffer die nachfolgenden drei Ebenen, auf welchen die Sprachenpolitik realisiert wird:

1) europäische Sprachenpolitik

2) regionale Sprachenpolitik ${ }^{4}$

3) polnische Sprachenpolitik.

Das von Pfeiffer für Europa und Polen vorgeschlagene Konzept, übertragen auf Asien im Allgemeinen und auf Japan im Besonderen, stellt sich folgendermaßen dar:

1) asiatische Sprachenpolitik

2) regionale Sprachenpolitik

3) japanische Sprachenpolitik.

Betrachtet man die höchste Ebene, d. h. Ebene Nummer 1 genauer, stellt man fest, dass zu den Prozessen, die im 21. Jahrhundert sowohl in Europa als auch in Asien implizite den Veränderungen in der Sprachenpolitik Vorschub leisteten, außer der Migration vor allem die Globalisierung gehört, verstanden als die zunehmende internationale Verflechtung im politischen, kulturellen und wirtschaftlichen Bereich. Die damit einhergehende Ausbreitung des Englischen in immer mehr Branchen steht in einem engen Zusammenhang mit der von S. Grucza (2009: 15) als rasant bezeichneten Entwicklung der fachlichen Spezialisierung der Welt, die sich vorrangig auf dem Wege der inter- und multikulturellen (Fach)Kommunikation vollzieht, die nicht erfolgen kann, „wenn jeder nur seine eigene Sprache spricht, sie kann sich auch nicht voll entfalten, wenn man nur eine ,große' Sprache spricht" (Pfeiffer 2005: 306-307). Wie dem Zitat entnommen werden kann, spricht sich Pfeiffer gegen die Dominanz einer Lingua franca aus, die heutzutage ohne jeden Zweifel die englische Sprache ist. Lingua franca, einst bezeichnet als Sprache der Franken, war eine mittelalterliche Verkehrssprache, welche in der Zeit der Kreuzzüge im östlichen Mittelmeerraum im Kontakt zwischen Christen und Moslems entstanden ist (vgl. Bußmann 2008: 408). Während frühere Verkehrssprachen wie beispielsweise das Lateinische, das Französische, das Russische in der ehemaligen Sowjetunion oder Swahili in Ostafrika nur in bestimmten Regionen der Welt verwendet wurden/werden, scheint das Englische als die erste Weltsprache, als globale Lingua franca in der Menschengeschichte anerkannt zu werden. Beim Beherrschen des Englischen und anderer Sprachen sind verschiedene Aspekte zu berücksich-

\footnotetext{
${ }^{4}$ Dabei handelt es sich um die Fremdsprachenpolitik in Grenzregionen, auf welche im vorliegenden Beitrag aus Platzgründen nicht näher eingegangen werden wird.
} 
tigen, je nachdem, ob diese Sprachen als Muttersprachen, als Zweitsprachen oder als Fremdsprachen erworben/gelernt werden. Für die Sprecher der englischen Sprache schlägt Crystal (2003: 61) in diesem Zusammenhang in Anlehnung an Kachru (1988: 5) folgendes Modell vor, das einen komplexen Bereich anhand einer einfachen Einteilung wie folgend strukturiert:

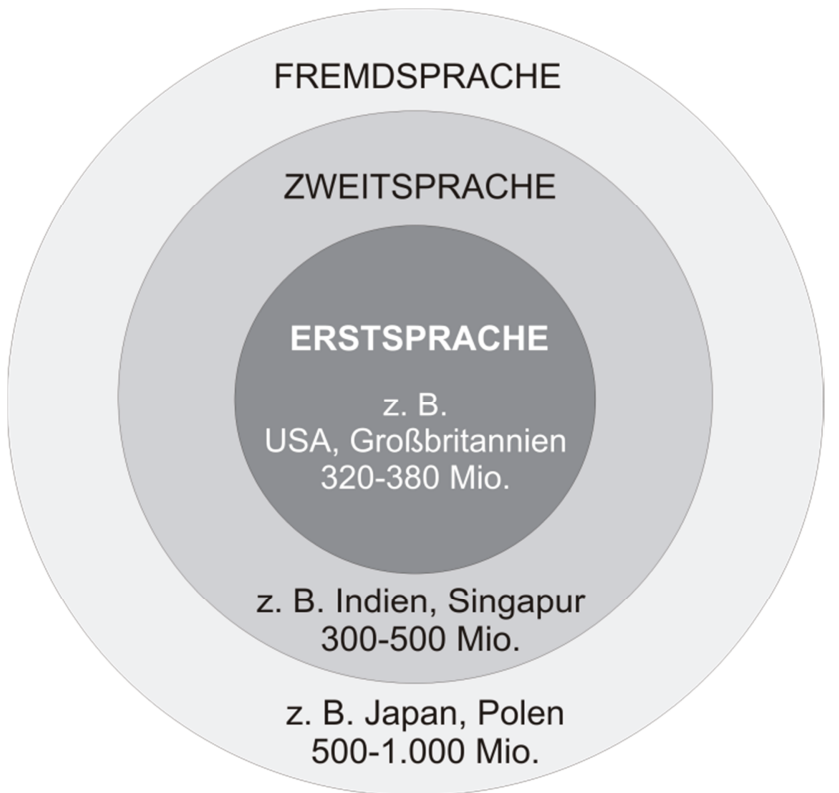

Abbildung 1. Die drei „Kreise” des Englischen (eigene Zusammenstellung auf der Grundlage von Crystal (2003: 61) in Anlehnung an Kachru (1988: 5))

Die Gemeinsamkeit der beiden hier untersuchten Länder bildet die Zugehörigkeit zu dem expandierenden Kreis.

\section{FREMDSPRACHENPOLITIK IN POLEN}

Es ist eingangs darauf hinzuweisen, dass in Polen sich zwei Ministerien mit der Bildungspolitik beschäftigen. Während die Bereiche Vorschule und Schule in die Zuständigkeit des Ministeriums für Nationale Bildung (Polnisch: Ministerstwo Edukacji Narodowej) fallen, sind Forschung und Hochschule im Aufgabenbereich des Ministeriums für Wissenschaft und Hochschulbildung (Polnisch: Ministerstwo Nauki i Szkolnictwa Wyższego) angesiedelt. 
Die Fremdprachenpolitik steht in einem engen Zusammenhang mit der Bildungspolitik, die sich in Polen ständig im Wandel befindet, da mehrere strukturelle Reformen seit der Wende 1989 durchgeführt wurden, denen auch der Vorschulbereich ausgesetzt ist. Gegenwärtig ist der Kindergarten in Polen als eine Einrichtung für Kinder im Alter von 3 (in Ausnahmefällen 2,5) bis 6 Jahren konzipiert, wobei das verpflichtende Vorschuljahr nur das 6 . Lebensjahr umfasst. Eingeschult werden die Kinder in der Regel mit sieben Jahren. Die neueste Schulreform, die hauptsächlich die Abkehr von der dreistufigen Schulform zu einer zweistufigen bekräftigt, trat am 1. September 2017 in Kraft. Das bisherige Basisschulsystem umfasste eine sechsjährige Grundschule (Polnisch: szkoła podstawowa), eine dreijährige Mittelschule (Polnisch: gimnazjum) und eine dreijährige Oberschule (Polnisch: liceum) mit Abitur. Die größte Strukturdifferenz zwischen dem alten und neuen System äußert sich somit in der Verlängerung der Grundschulzeit von sechs auf acht Jahre. Die nunmehr achtjährige Grundschule wurde dabei in zwei Stufen unterteilt: erste (Jahrgangsstufen eins bis drei) und zweite (Jahrgangsstufen vier bis acht). Des Weiteren wurde die bisherige Mittelstufe aufgelöst, die Klassen sieben bis neun abdeckte. Anstelle einer dreijährigen Oberschule entstand eine vierjährige Oberschule. Die Schulpflicht beginnt mit dem 7. Lebensjahr und gilt bis zum 18. Lebensjahr.

Die Fremdsprachenpolitik Polens steht darüber hinaus in einem engen Zusammenhang mit der europäischen Sprachenpolitik, die für Mehrsprachigkeit plädiert. Das Thema der Mehrsprachigkeit gewann besonders im Verlauf der 1990er Jahre auf europäischer Ebene an Bedeutung. Im „Charta der europäischen Identität" vom Jahre 1995, deren Entstehung durch den ehemaligen Präsidenten der Tschechischen Republik Václav Havel angeregt wurde, wird den europäischen Bürgern empfohlen, Mehrsprachigkeit zu erwerben. Die von der EU offiziell geförderte Mehrsprachigkeit nimmt in der Praxis der Mitgliedsländer vor allem folgende Form an: das Modell 1+2 (am häufigsten als Muttersprache plus Englisch plus eine weitere Fremdsprache aufgefasst). In Polen werden die Fragen des Fremdsprachenunterrichts in den Verordnungen des Ministers für Nationale Bildung über die Programmgrundlagen geregelt. Dabei handelt es sich um folgende Verordnungen:

1) Die Verordnung des Ministers für Nationale Bildung vom 14. Februar 2017 über die Programmgrundlage der Vorschul- und Allgemeinbildung in der Grundschule (...).

2) Die Verordnung des Ministers für Nationale Bildung vom 30. Januar 2018 über die Programmgrundlage der Allgemeinbildung in der Oberschule (...).

Fremdsprachen haben in Polen bereits den Eingang in die Programmgrundlage der Vorschulbildung gefunden. Die Idee, die dahinter steckt, ba- 
siert auf der Annahme, dass Kinder im Vorschulalter für eine der neueren Sprachen und andere Kulturen sensibilisiert werden sollen. Die in die Bildungsarbeit eingebaute Begegnung mit der Sprache soll auf eine spielerische Art und Weise erfolgen und sich vor allem auf Alltagssituationen beziehen. Obwohl in dem Dokument keine konkrete neuere Sprache angegeben wurde, soll den Kindern im Anschluss an die Kindergartenzeit die Möglichkeit gegeben werden, weiter dieselbe Sprache in der Grundschule zu lernen.

In Polen gibt es ein vom Ministerium für Nationale Bildung erarbeitetes Konzept, welches Fremdsprachenunterricht an polnischen Grundschulen voraussetzt, sowie des Weiteren ebenso das von der EU geförderte Model $1+2$ in die Tat umsetzt, indem zugleich zwei Fremdsprachen in die Reihe der Pflichtfächer aufgenommen werden sollen, wenn auch nicht gleich ab der ersten Klasse. Eine Übersicht über Details soll folgende Tabelle geben:

Tabelle 1. Eigene Zusammenstellung auf der Grundlage der Verordnung des Ministers für Nationale Bildung über die Programmgrundlage vom 14. Februar 2017 sowie der Programmgrundlage für moderne Fremdsprachen in Grundschulen mit jeweiligem Kommentar

\begin{tabular}{|c|c|c|c|c|}
\hline Bildungsetappe & $\begin{array}{c}\text { Fremdsprache } \\
\text { unterrichtet } \\
\text { als... }\end{array}$ & Beschreibung & $\begin{array}{c}\text { Anzahl der } \\
\text { Stunden pro } \\
\text { Schuljahr }\end{array}$ & $\begin{array}{c}\text { Sprachniveaustufe nach } \\
\text { dem Gemeinsamen } \\
\text { Europäischen Referenz- } \\
\text { rahmen für Sprachen }\end{array}$ \\
\hline $\begin{array}{c}\text { I } \\
\text { Grundschule } \\
\text { Primarbereich } \\
\text { Jahrgangsstufen } \\
1-3\end{array}$ & erste & $\begin{array}{c}\text { ab der ersten } \\
\text { Klasse }\end{array}$ & 180 & $\mathrm{~A} 1$ \\
\hline $\begin{array}{c}\text { II } \\
\text { Grundschule } \\
\text { Jahrgangsstufen } \\
4-8\end{array}$ & erste & $\begin{array}{c}\text { Fortführung } \\
\text { des Unterrichts }\end{array}$ & 450 & A2+ \\
\cline { 2 - 5 } & zweite & $\begin{array}{c}\text { ab der siebten } \\
\text { Klasse }\end{array}$ & 120 & A1 \\
\hline
\end{tabular}

Bereits Erstklässler lernen eine Fremdsprache, zwar nicht isoliert, sondern im Rahmen eines für die Jahrgangsstufen 1-3 vorgesehenen integrierten Konzepts, welches sich in die Begegnung mit der Fremdsprache didaktisch und methodisch einfügt. Dieser Bildungsetappe liegt zudem die Idee zugrunde, dass Fremdsprachenlernen nicht als das Ziel an sich betrachtet werden sollte. Vielmehr handelt es sich dabei um eine vielseitige Entwicklung des Kindes, die verschiedene Aspekte umfasst, welche $u$. a. die Freude an der Beschäftigung mit fremder Sprache fördert, was wiederum zu einer offenen und toleranten Einstellung der Sprachen- sowie Kulturenvielfalt 
führt. Eine weitere wichtige Grundlage des Fremdsprachenunterrichts in den nächsten Bildungsstufen bildet der Aufbau der kommunikativen Kompetenz, aus diesem Grund ist es von großem Belang den Lehrprozess so zu steuern, dass die Schüler/-innen die Überzeugung gewinnen, Fremdsprachenerlernung sei nützlich oder sogar notwendig für die Verwirklichung der eigenen Kommunikationsabsichten im Alltag.

Es ist darüber hinaus auf die Tatsache hinzuweisen, dass ab der 7. Klasse noch die zweite Fremdsprache hinzukommt, welche aber wiederum in einem kleineren Umfang unterrichtet wird. Es wird im polnischen Bildungssystem das von der EU geförderte Model 1+2 in die Tat umgesetzt. Da im Fremdsprachenunterricht der Kontinuität eine Schlüsselrolle zukommt, wird laut neuester Richtlinien des Ministeriums für Nationale Bildung die Fortführung der 1. Pflichtsprache nicht nur in der zweiten Bildungsetappe, sondern auch in der dritten Bildungsetappe gewährleistet werden, sodass sich die Lerndauer je nach Schultyp auf 12 bzw. 13 Jahre erstreckt. Empfohlen wird dieses Modell auch für die zweite Fremdsprache, wenn auch nicht so eindeutig wie für die erste. Die weiterführenden informierenden Details über den Fremdsprachenunterricht in der Oberschule sind der folgenden Tabelle zu entnehmen:

Tabelle 2. Eigene Zusammenstellung auf der Grundlage der Verordnung des Ministers für Nationale Bildung über die Programmgrundlage vom 30. Januar 2018

\begin{tabular}{|c|c|c|c|c|}
\hline Bildungsetappe & $\begin{array}{c}\text { Neuere } \\
\text { Fremdsprache } \\
\text { unterrichtet als }\end{array}$ & Beschreibung & $\begin{array}{c}\text { Anzahl der } \\
\text { Stunden pro } \\
\text { Schuljahr }\end{array}$ & $\begin{array}{c}\text { Sprachniveaustufe } \\
\text { nach dem Gemein- } \\
\text { samen Europäischen } \\
\text { Referenzrahmen für } \\
\text { Sprachen }\end{array}$ \\
\hline \multirow{4}{*}{$\begin{array}{c}\text { III } \\
\text { Oberschule }\end{array}$} & \multirow{2}{*}{ erste } & $\begin{array}{c}\text { Fortführung des } \\
\text { Unterrichts } \\
\text { (Grundstufe) }\end{array}$ & 360 & $\begin{array}{c}\text { B1+ } \\
\text { (B2 Rezipieren von } \\
\text { Äußerungen) }\end{array}$ \\
\cline { 2 - 5 } & $\begin{array}{c}\text { Fortführung des } \\
\text { Unterrichts } \\
\text { (erweiterte Stufe) }\end{array}$ & $360+180=540$ & $\begin{array}{c}\text { B2+ } \\
\text { (C1 Rezipieren von } \\
\text { Äußerungen) }\end{array}$ \\
\cline { 2 - 5 } & zweite & $\begin{array}{c}\text { Fortführung des } \\
\text { anterrichts aus der } \\
\text { Grundschule }\end{array}$ & 240 & A2 \\
\cline { 2 - 5 } & & \multicolumn{2}{|c|}{ A2+ } \\
\hline
\end{tabular}

Es wird darauf hingewiesen, dass momentan bedauerlicherweise noch keine Programmgrundlage für moderne Fremdsprachen an Oberschulen mit jeweiligem Kommentar vorliegt. Im polnischen Bildungssystem wird in der 
dritten Bildungsetappe auf das Modell 1+2 zurückgegriffen. In diesem Bildungsmodell werden zwei moderne Fremdsprachen in die Pflichtfächerreihe mit aufgenommen.

Die Fragen des Fremdsprachenunterrichts an Hochschulen werden u. a. in der Verordnung des Ministers für Wissenschaft und Hochschulbildung vom 2. November 2011 über den Nationalen Qualifikationsrahmen für Hochschulbildung geregelt. Der Nationale Qualifikationsrahmen (im Weiteren: NQR) ist als nationale Umsetzung des Europäischen Qualifikationsrahmens zu verstehen, welcher wiederum zur Vergleichbarkeit polnischer Qualifikationen in Europa beisteuert. Der NQR basiert auf folgenden drei Säulen auf dem Wissen, den Fertigkeiten und der Sozialkompetenz, um die im polnischen Hochschulsystem angestrebten Lernergebnisse angemessen darzustellen. In dem Dokument wurden Fremdsprachenkenntnisse dem Bereich der Fertigkeiten zugeordnet. Dabei handelt es sich lediglich um die beiden produktiven Sprachfertigkeiten, d. h. das Schreiben und das Sprechen. Die vorgegebenen Anforderungen fasst die folgende unten aufgeführte Tabelle zusammen:

Tabelle 3. Eigene Zusammenstellung auf der Grundlage der Verordnung des Ministers für Wissenschaft und Hochschulbildung vom 2. November 2011

\begin{tabular}{|l|l|l|}
\hline \multicolumn{1}{|c|}{ Studienzyklus } & \multicolumn{1}{|c|}{ Bachelor } & \multicolumn{1}{c|}{ Master } \\
\hline Sprache (-n) & $\begin{array}{l}\text { (Polnisch) und eine für die } \\
\text { jeweilige Wissenschaftsdisziplin } \\
\text { typische Fremdsprache }\end{array}$ & $\begin{array}{l}\text { (Polnisch) und eine für die je- } \\
\text { weilige Wissenschaftsdisziplin } \\
\text { typische Fremdsprache }\end{array}$ \\
\hline Schreiben & typische Texte verfassen & komplexe Texte verfassen \\
\hline Sprechen & Präsentationen vortragen & $\begin{array}{l}\text { systematisch angelegte Präsenta- } \\
\text { tionen vortragen }\end{array}$ \\
\hline $\begin{array}{l}\text { Sprachniveaustufe nach } \\
\text { dem Gemeinsamen } \\
\text { Europäischen Referenz- } \\
\text { rahmen für Sprachen }\end{array}$ & B2 & \\
\hline
\end{tabular}

Im Gegensatz zu früheren Bildungsetappen, auf denen bis auf die ersten sechs Jahre der Grundschule, vorwiegend das Modell 1+2 zum Einsatz kommt, kann man im Kontext polnischer Hochschulbildung vom Modell 1+1 sprechen. Hinsichtlich der Auswahl einer bestimmten Fremdsprache stehen den Student/-innen unterschiedliche Möglichkeiten zur Verfügung (so werden beispielsweise Englisch, Deutsch, Französisch, Russisch, Spanisch, Italienisch oder Latein an der Adam-Mickiewicz-Universität Posen angeboten). 


\section{FREMDSPRACHENPOLITIK IN JAPAN}

Japan gilt als ein überaus hoch entwickeltes Industrieland, in dem das permanente Bemühen um Perfektionierung in allen Lebensbereichen eine Selbstverständlichkeit darstellt (man denke beispielsweise an das im Westen übernommene Kaizen-Konzept, in dessen Mittelpunkt das Streben nach kontinuierlicher Verbesserung steht). So mag es nicht sehr verwunderlich sein, dass in der japanischen Regierung das Thema der Erlernung bzw. Verbesserung von Fremdsprachenkenntnissen der Bevölkerung zur Diskussion gestellt wird. Den Ausgangspunkt der Debatte zum Ende des 20. Jahrhunderts bildeten die Ergebnisse der TOEFL-Prüfungen vom Jahre 1998, bei welchen die Japaner am schlechtesten in Asien abgeschnitten haben. 1999 wies der Ministerpräsident Keizō Obuchi darauf hin, dass „(...) English should be the official second language of Japan and this attracted a lot of debate in the following year" (Tada 2016: 25). Zur Jahrhundertwende wurde ein ausführlicher (246 Seiten) Report der japanischen Ministerkommission Japan's Goals in the 21th Century (2000) entworfen, welcher wiederum als eine zukunftsorientierte, mehrdimensionale Konzeption des bestmöglichen Lebens und der Weiterentwicklung der japanischen Gesellschaft anzusehen ist. In dem erwähnten Dokument wurde nicht nur deutlich für "the mastery of English as the international lingua franca" (Kap. 1, S. 17) plädiert, sondern auch über den möglichen Status des Englischen in Japan diskutiert.

So long as English is effectively the language of international discourse, there is no alternative to familiarizing ourselves with it within Japan. Even if we stop short of making it an official second language, we should give it the status of a second working language and use it routinely alongside Japanese.

Im Anschluss an den Report erfolgte drei Jahre später der Aktionsplan Regarding the Establishment of an Action Plan to Cultivate Japanese with English Abilities (2003). Die damalige Ministerin Atsuko Toyama setzte sich in dem Bericht einerseits mit den Herausforderungen der Globalisierung in Japan an sich, sowie der überaus großen Rolle der englischen Sprache im Globalisierungsprozess andererseits, auseinander:

In such a situation, English has played a central role as the common international language in linking people who have different mother tongues. For children living in the 21st century, it is essential for them to acquire communication abilities in English as a common international language. In addition, English abilities are important in terms of linking our country with the rest of the world, obtaining the world's understanding and trust, enhancing our international presence and further developing our nation. At present, though, due to the lack of sufficient ability, many Japanese are restricted in their exchanges with foreigners and 
their ideas or opinions are not evaluated appropriately. It is also necessary for Japanese to develop their ability to clearly express their own opinions in Japanese first in order to learn English.

Die zitierten Erläuterungen lassen eine bestimmte Entwicklung erkennen, bei der sowohl die kommunikative Kompetenz in der englischen Sprache als auch die Fähigkeit, sich im Japanischen adäquat zu äußern, in den Vordergrund rücken. Die Fähigkeit der Menschen, ihre Gedanken zuerst klar in der Muttersprache formulieren zu können, scheint eine wichtige Voraussetzung dafür zu sein, später die Fremdsprache Englisch „as a common international language" zu lernen. Ausreichende Sprachkenntnisse sind insofern von groBer Relevanz, als erst sie es den Japanern ermöglichen, an den „Rest der Welt" angeschlossen zu sein und die internationale Präsenz Japans zu stärken. Fehlen indessen die entsprechenden Kenntnisse, besteht die Gefahr, dass es zu Missverständnissen kommen kann. Um das Ziel der Entwicklungsvertiefung von Englischkenntnissen der japanischen Bevölkerung zu erreichen, wurde 2014 anhand einer Reform mit dem Programm unter dem Namen English Education Reform Plan Corresponding to Globalization (MEXT, 2014) angefangen. Die Abkürzung MEXT steht für das Mombukagakushō (Ministerium für Bildung, Kultur, Sport, Wissenschaft und Technologie). Im Gegensatz zu Polen ist diese für die Bildungspolitik in allen Stufen zuständig. Die thematischen Schwerpunkte des Ministeriums werden durch einige Abteilungen abgebildet, von denen sich vier direkt mit der Bildung befassen, diese werden wie folgend dargestellt:

1) Elementary and Secondary Education Bureau (Early Childhood Education Division inbegriffen)

2) Higher Education Bureau

3) Lifelong Learning Policy Bureau

4) Private Education Institution Department

Vor dem eigentlichen Schuleintritt können die Kinder in Japan zuerst eine Kinderkrippe und danach einen Kindergaten besuchen, welcher für die Altersstufe von 3-5 Jahren vorgesehen ist. Hinzuweisen ist dabei, dass der Besuch der Vorschule freiwillig und daher auch kostenpflichtig ist. Da der Fremdsprachenunterricht in Japan im Vorschulbereich im Allgemeinen staatlich nicht geregelt wird, beginnt dieser erst offiziell in der Grundschule.

Das oft als 6-3-3-4-System bezeichnete Basisschulsystem Japans umfasst eine sechsjährige Grundschule (Japanisch: shōgakkō), eine dreijährige Mittelschule (Japanisch: chūgakkō), eine dreijährige Oberschule (Japanisch: kōtō gakkō) und eine Hochschule (Japanisch: daigaku), welche mit dem Abschluss des Bachelors (4 Jahre) absolviert wird. Das Studium an einer japanischen Universität kann weiter fortgesetzt werden. Es können die Abschlüsse zum 
einen im Master (2 Jahre) und zum anderen im Doktorat (3 Jahre) gemacht werden.

Die Grundschule umfasst die jeweiligen Altersgruppen von sechs bis zwölf Jahren. Mit dem Abschluss der Mittelstufe, welche im Allgemeinen mit 15 Jahren erreicht wird, endet zugleich auch die Schulpflicht. Es ist dabei doch zu erwähnen, dass ein Großteil der Schüler/-innen weiterführende Schulen wie z. B. Oberschulen besucht.

Die Begegnung mit der englischen Sprache (als „English Language Activities" bezeichnet), beginnt in der Jahrgangsstufe 3 (vorher war der Beginn in der Jahrgangsstufe 5, welche wiederum vom Klassenlehrer geführt wurde). Für die Jahrgangsstufen 3 und 4 sind 1-2 Stunden pro Woche vorgesehen. Als das wichtigste Ziel der Aktivitäten, nach deren flächendeckender Einführung, fungiert der Aufbau des grundlegenden Sprachkönnens. Erst der für die Jahrgangsstufen 5 und 6 geplante Unterricht hat einen offiziellen Status als Schulfach, welcher oftmals dreimal pro Woche stattfinden sollte. Im Mittelpunkt des Unterrichts, der in dieser Stufe von zwei Lehrern (Klassenlehrer mit Englischkenntnissen sowie einer höherstehenden pädagogischen Kompetenz und einem Fachlehrer für Englisch) erteilt wird, steht die Förderung von Basiskompetenzen im Englischen im Vordergrund. Im Unterschied zu den Richtlinien für Grundschulen, wird in denen für Mittelschulen weder die vorgesehene Anzahl der Unterrichtsstunden noch die der Lehrkräfte angegeben. Präzisiert wird indessen die Unterrichtssprache, d. h. das Englische, diese stellt wiederum die Basis dar, auf welcher der Unterricht in den Jahrgangsstufen 7-9 im Prinzip angestrebt werden soll. Ebenso in der Oberschule soll der Unterricht auf englischem Sprachniveau geführt werden. Zum Einsatz kommen sog. high-linguistic activities wie beispielsweise (Präsentationen, Debatten sowie Verhandlungen).

Da der Reformplan einen überaus allgemeinen Charakter hat, werden viele Details nicht präzisiert (zumindest in der mir zur Verfügung stehenden englischen Version). Als die der Reform zugrunde liegenden Hauptziele werden folgende zwei aufgeführt:

1) To ensure nurturing English communication skills establishing coherent learning achievement targets throughout elementary and lower/upper secondary school.

2) Enrich educational context in relation to nurturing individual's sense of Japanese identity (focus on traditional culture and history among other things).

Während hingegen der erste Punkt eindeutig formuliert worden ist und sich hauptsächlich auf die Entwicklung kommunikativer Kompetenzen der Schüler/-innen konzentriert, erweist sich der zweite Punkt wiederum auf den ersten Blick als ziemlich vage. Es bleibt unklar, wie Englischlehr/-innen zur Entfaltung der japanischen Identität beitragen können. Sicherlich ist die 
auf der Entwicklung des sprachlichen Könnens basierende pragmatische Dimension dominierend. Laut dieser These wird das Englische als ein besonderes Kommunikationsmittel betrachtet, welches somit keine bestimmte Zielkultur bzw. Kulturwerte vertritt.

Von der besprochenen Reform sind Hochschulen ausgeschlossen worden. Allerdings sind die Fragen des Fremdsprachenunterrichts an Hochschulen in Japan insofern von großer Relevanz, als der Zugang zu einer japanischen Hochschule durch eine zentrale Aufnahmeprüfung geregelt wird, welche obligatorisch in den Fächern Englisch, Japanisch und Mathematik abgelegt werden. Es ist zu erwähnen, dass Englisch die einzige Fremdsprache ist, die als Prüfungsfach angeboten wird. Laut neuesten Richtlinien des MEXT (vom 13. Juli 2017) wird die Form der Englischprüfung in den nächsten Jahren einer großen Reform unterzogen. Anstelle der derzeit verwendeten Multiple-Choice Prüfung, die lediglich auf das Testen einer rezeptiven Sprachfertigkeit, d. h. des Leseverstehens und einer produktiven Sprachfertigkeit, d. h. des Schreibens beschränkt wurde, werden die von privater Seite gehandhabten Prüfungen wie beispielsweise TOEIC (Test of English for International Communication), eingesetzt. Im Rahmen der neuen Prüfung werden daher alle vier Fertigkeiten getestet und bewertet. Den Schüler/-innen im letzten Oberschuljahr wird die Möglichkeit angeboten, zwischen April und Dezember bis zu zwei Mal an den Englischprüfungen teilnehmen zu können, deren Ergebnisse im Namen der Schüler/-innen an die von ihnen ausgewählte Universität weitergeleitet werden. Die Prüfungen werden in keinen anderen Sprachen angeboten.

Bis zum Jahre 1991 wurden in Japan zwei Fremdsprachen in der Grundausbildung (den ersten zwei Jahren des Studiums) an den Hochschulen obligatorisch angeboten, was doch wiederum im Rahmen der Reform des Hochschulgründungsgesetzes abgeschafft wurde. An vielen Hochschulen in Japan ist demzufolge das Erlernen einer zweiten Fremdsprache keine Verpflichtung mehr. Da Hochschulen über Autonomie in diesem Bereich verfügen, hängt die Anzahl der Unterrichtsstunden von der jeweiligen Universität sowie der jeweiligen Fakultät ab. Die erste Wahl fällt meist auf die englische Sprache - wird dennoch eine zweite Fremdsprache belegt - werden vor allem asiatische Fremdsprachen gewählt (vgl. Fujiwara 2013: 33).

\section{FAZIT}

Greift man die eingangs aufgestellten Leitfragen wieder auf, so lässt sich feststellen, dass der allgemeine Trend in beiden untersuchten Ländern in die Richtung des frühzeitigen Spracherwerbs geht. In Polen beginnen die 
Schüler/-innen früher mit dem Erlernen einer Sprache, spätestens jedoch ab dem 6. Lebensjahr, d. h. im verpflichtenden Vorschuljahr. In Japan wiederum werden die linguistischen Aktivitäten in der Schule für Drittklässler angeboten, d. h. im 8. Lebensalter. Es ist darauf hinzuweisen, dass darüber hinaus in der polnischen Grundschule (7. Schulklasse) eine zweite Fremdsprache zum alltäglichen Lernprogramm der Schüler/-innen hinzukommt, wodurch das Modell 1+2 verwirklicht wird. Die japanische Fremdsprachenpolitik zielt dagegen eindeutig auf die Förderung der englischen Sprache ab, welche als einzige Fremdsprache an Grund-, Mittel- und Oberschulen flächendeckend angeboten wird. Hinsichtlich der Auswahl einer bestimmten Fremdsprache bzw. bestimmter Sprachenfolge stehen den polnischen Schüler/-innen theoretisch unterschiedliche Möglichkeiten zur Verfügung. In den analysierten Dokumenten ist die Rede von einer bzw. mehreren neueren / modernen Fremdsprachen (Polnisch: język obcy nowożytny). Dabei fällt auf, dass das Englische nirgendwo explizite genannt wird, auch wenn es momentan die erste obligatorische Fremdsprache in Europa ist. Die Auswahl der zu erlernenden Sprachen, je nach Universität, haben japanische Schüler/-innen erst in der Hochschulbildung. In der nachfolgend aufgeführten Tabelle werden jeweils die wichtigsten Unterschiede der beiden Länder zusammenfassend dargestellt:

Tabelle 4. Die wichtigsten Unterschiede im Fremdsprachenunterricht Polens und Japans (eigene Zusammenstellung)

\begin{tabular}{|l|l|l|}
\hline \multicolumn{1}{|c|}{ Bildungsetappe } & \multicolumn{1}{|c|}{ Polen } & \multicolumn{1}{c|}{ Japan } \\
\hline Kindergarten & $\begin{array}{l}\text { die erste Begegnung mit der } \\
\text { Sprache }\end{array}$ & staatlich nicht geregelt \\
\hline Grundschule & $\begin{array}{l}\text { ab der ersten Klasse eine Fremd- } \\
\text { sprache obligatorisch, in der } \\
\text { Regel mit 7 Jahren, werden } \\
\text { mindestens 3 Stunden pro Woche } \\
\text { angeboten }\end{array}$ & $\begin{array}{l}\text { ab der dritten Klasse Englisch } \\
\text { obligatorisch, mit 8 Jahren, wer- } \\
\text { den mindestens 1-2 Stunden pro } \\
\text { Woche angeboten } \\
\text { ab der fünften Klasse, 3 Stunden } \\
\text { pro Woche }\end{array}$ \\
\hline $\begin{array}{l}\text { Grundschule/Mittel- } \\
\text { schule }\end{array}$ & $\begin{array}{l}\text { ab der siebten Klasse zwei } \\
\text { Fremdsprachen obligatorisch }\end{array}$ & $\begin{array}{l}\text { ab der siebten Klasse Englisch in } \\
\text { der Unterrichtssprache Englisch }\end{array}$ \\
\hline Oberschule & $\begin{array}{l}\text { zwei Fremdsprachen obligato- } \\
\text { risch }\end{array}$ & $\begin{array}{l}\text { der Englischunterricht auf engli- } \\
\text { schem Sprachniveau }\end{array}$ \\
\hline Hochschule & $\begin{array}{l}\text { eine Fremdsprache obligatorisch } \\
\text { Bachelor: das Niveaustufe B2 } \\
\text { soll erreicht werden } \\
\text { Master: das Niveaustufe B2+ soll } \\
\text { erreicht werden }\end{array}$ & $\begin{array}{l}\text { eine zentrale Aufnahmeprüfung } \\
\text { im Fach Englisch } \\
\text { eine bzw. zwei Fremdsprachen } \\
\text { in den ersten zwei Jahren des } \\
\text { Studiums (je nach Universität) }\end{array}$ \\
\hline
\end{tabular}


$\mathrm{Zu}$ den Schwerpunkten der Lehrprogramme in beiden untersuchten Ländern lässt sich in einer kurzen Auswertung abschließend Folgendes feststellen: Der Fremdsprachenunterricht ist das gemeinsame Hauptziel von Polen sowie von Japan. Es soll dabei auf das sprachliche Können fokussiert werden, welches wiederum der Kommunikationsfähigkeit eine enorm große Bedeutung zukommen lässt. Es ist weiterhin zu erwähnen, dass in polnischen Richtlinien die wechselseitige Verflechtung von Sprache und Kultur spezifisch zum Ausdruck gebracht wird, deren Zusammenhang in der modernen Fremdsprachendidaktik hervorgehoben wird.

\section{LITERATURVERZEICHNIS}

Ammon, U. (2010). Sprachenpolitik. In: H. Glück (Hrsg.), Metzler Lexikon Sprache (S. 636). Stuttgart und Weimar: Verlag J.B. Metzler.

Bochmann, K. (1993). Theorie und Methoden der Sprachpolitik und ihrer Analyse. In: K. Bochmann (Hrsg.), Sprachpolitik in der Romania: Zur Geschichte sprachpolitischen Denkens und Handelns von der Französischen Revolution bis zur Gegenwart (S. 3-62). Berlin und New York: Walter de Gruyter.

Bußmann, H. (2002). Sprach(en)politik. In: H. Bußmann (Hrsg.), Bußmann Lexikon Sprachwissenschaft (S. 628). Stuttgart: Kröner.

Bußmann, H. (2008). Lingua franca. In: H. Bußmann (Hrsg.), Bußmann Lexikon Sprachwissenschaft (S. 408). Stuttgart: Kröner.

Crystal, D. (2003). English as a global language. Cambridge: Cambridge University Press.

Fujiwara, M. (2013). Motivation japanischer Deutschlernender im universitären Bereich - Eine longitudinale Studie im Anfängerniveau nach der Selbstbestimmungstheorie von Deci \& Ryan. Neue Beiträge zur Germanistik 12 (1), 33-52.

Grucza, S. (2009). Fachwissen-Fachsprache-Fachdiskurs: Fachdiskursanalyse aus der Sicht der anthropozentrischen Sprachentheorie. In: F. Grucza / G. Pawłowski / R. Utri (Hrsg.), Diskurse als Mittel und Gegenstände der Germanistik. Materialien der Jahrestagung und internationalen wissenschaftlichen Konferenz des Verbandes Polnischer Germanisten abgehalten an der Warmia und Mazury Universität, 8-10. Mai 2009, Olsztyn (S. 15-28). Warszawa: EuroEdukacja.

Kachru, B. (1988). The sacred cows of English. English Today 16, 3-8.

Kirkpatrick, A., Liddicoat, A. J. (2017). Language education policy and practice in East and Southeast Asia. Language Teaching, 50 (2), 155-188. DOI: 10.1017/S0261444817000027.

Krumm, H. J. (2010). Sprachenpolitik. In: H. Barkowski / H. J. Krumm (Hrsg.), Fachlexikon Deutsch als Fremd- und Zweitsprache (S. 298). Tübingen und Basel: A. France Verlag.

Pfeiffer, W. (2004). Aktualne zagadnienia i wyzwania dla polskiej polityki językowej. In: I. Bartoszewicz / M. Hałub / A. Jurasz / (Hrsg.), Werte und Wertungen. Sprach-, Literaturund kulturwissenschaftliche Skizzen und Stellungnahmen. Festschrift für Eugeniusz Tomiczek zum 60. Geburtstag (S. 188-193). Wrocław: Oficyna Wydawnicza ATUT - Wrocławskie Wydawnictwo Oświatowe.

Pfeiffer, W. (2005). Gedanken zu polnischen und europäischen Sprachenpolitik und Integration. In: E. C. van Leewen (Hrsg.), Sprachenlernen als Investition in die Zukunft. Wirkungskreise 
eines Sprachlernzentrums. Festschrift für Heinrich P. Kelz zum 65. Geburtstag (S. 305-315). Tübingen: Gunter Narr Verlag.

Stark, F. (1997). Stationen deutscher Sprachenpolitik. In: S. Sporrer / M. Weber (Hrsg.), Sprachenpolitik in Europa - Sprachenpolitik für Europa (S. 27-38). Stuttgart: Institut für Auslandsbeziehungen.

Tada, M. (2016). Recent reform to the English education system in Japan. 21 ${ }^{\text {st }}$ Century Education Forum 11, 21-29.

Witt, J. (2001). Wohin steuern die Sprachen Europas? Probleme der EU-Sprachpolitik. Tübingen: Stauffenburg Verlag (ZAA Studies, 13).

\section{INTERNETQUELLEN}

Japanese Ministry of Education, Culture, Sports, Science and Technology (MEXT) (2003). Regarding the Establishment of an Action Plan to Cultivate "Japanese with English Abilities". http://www.gifunet.ed.jp/kyoka/eigo/CommunicativeEnglish/Regarding\%20the\%20Es tablishment $\% 20$ of $\% 20$ an $\% 20$ Action $\% 20$ Plan $\% 20$ to $\% 20$ Cultivate $\% 20 \%$ A1\%C8Japanese $\% 20$ with\%20English\%20Abilities\%A1\%C9.htm (Zugriff am: 24.04.2018).

Japanese Ministry of Education, Culture, Sports, Science and Technology (MEXT). http://www. mext.go.jp/en/ (Zugriff am: 20.04.2018).

Japanese Ministry of Education, Culture, Sports, Science and Technology (MEXT) (2014). English education reform plan corresponding to globalization.http://www.mext.go.jp/en/news/ topics/detail/1372656.htm (Zugriff am: 24.04.2018).

Japan's Goals in the 21th Century (2000). https://www.kantei.go.jp/jp/21century/report/ pdfs/index.html (Zugriff am: 22.03.2018).

Podstawa programowa kształcenia ogólnego z komentarzem. Szkoła podstawowa. Język obcy nowożytny (2017). https://cke.gov.pl/images/_EGZAMIN_OSMOKLASISTY/Podstawa_programowa/ SP_PP_2017_Jezyk_obcy_nowozytny.pdf (Zugriff am: 20.04.2018).

Rozporządzenie Ministra Edukacji Narodowej z dnia 14 lutego 2017 r. w sprawie podstawy programowej wychowania przedszkolnego oraz podstawy programowej kształcenia ogólnego dla szkoły podstawowej, w tym dla uczniów z niepełnosprawnością intelektualną w stopniu umiarkowanym lub znacznym, kształcenia ogólnego dla branżowej szkoły I stopnia, kształcenia ogólnego dla szkoły specjalnej przysposabiającej do pracy oraz kształcenia ogólnego dla szkoły policealnej (2017). http://dziennikustaw.gov.pl/du/ 2017/356/1 (Zugriff am: 20.04.2018).

Rozporządzenie Ministra Edukacji Narodowej z dnia 30 stycznia 2018 r. w sprawie podstawy programowej kształcenia ogólnego dla liceum ogólnokształcącego, technikum oraz branżowej szkoły II stopnia (2018). http:/ / www.dziennikustaw.gov.pl/DU/2018/467 (Zugriff am: 20.04.2018).

Rozporządzenie Ministra Nauki i Szkolnictwa Wyższego z dnia 2 listopada 2011 r. w sprawie Krajowych Ram Kwalifikacji dla Szkolnictwa Wyższego (2011). http://prawo.sejm.gov. pl/isap.nsf/download.xsp/ WDU20112531520 /O/D20111520.pdf (Zugriff am: 20.04.2018).

Received: 26.05.2018; revised: 3.03.2019 